ORIGINAL ARTICLE

\title{
Assessment of Sleep Quality Among Women of Childbearing Age by Pittsburgh Sleep Quality Index
}

\author{
Anshuman Pattanaik ${ }^{1}$, Arpita Priyadarshini ${ }^{2}$, Sameer Vaidya $^{3}$, Nupur Pattanaik ${ }^{4}$
}

\begin{abstract}
Background: Complaints of sleep disturbance are more prevalent among women. Women in childbearing age (15-49 years) are prone to sleep disturbances due to the menstrual cycle, pregnancy, and many other hormonal changes. So this study was designed with an objective to assess the sleep quality among women of childbearing age.

Materials and methods: This cross-sectional study was conducted in sleep disorder clinic and laboratory of Srirama Chandra Bhanja Medical College, Cuttack, Odhisa, India between October 2014 and September 2015. Two hundred eleven women of childbearing age participated. Sleep was assessed by the Pittsburgh sleep quality index (PSQI) scale.

Results: Among poor sleeper women $56.5 \%$ were from age 15 to 26 years which is higher from other age groups. About $61.9 \%$ of pregnant women were poor sleepers. About $83.3 \%$ of the second-trimester pregnant women were poor sleepers.

Conclusion: Sleep abnormality in women of childbearing age starts from a very early age. Most pregnant women suffer from sleep abnormality mainly in the second trimester.
\end{abstract}

Keywords: Pittsburgh sleep quality index, Pregnancy, Sleep quality, Women.

Indian Journal of Sleep Medicine (2019): 10.5005/jp-journals-10069-0032

\section{INTRODUCTION}

ufficient sleep is essential for immunocompetence ${ }^{1}$ and for overall health. ${ }^{2}$ Complaints of sleep disturbance are more prevalent among women than men across the entire lifespan., Women in childbearing age (15-49years) ${ }^{5}$ are prone to sleep disturbances due to the menstrual cycle, pregnancy, and many other hormonal changes. ${ }^{6}$ A decrease in sleep leads to an increase in inflammatory cytokines which are now believed to be important in the development of health problems. ${ }^{7}$ This study was done with an objective to assess the sleep quality among the women of childbearing age.

\section{Materials AND Methods}

This cross-sectional study was conducted at a sleep disorder clinic in the Department of Physiology, Srirama Chandra Bhanja Medical College, Cuttack, Odisha between October 2014 and September 2015. Those women who gave written consent to participate were included in the study. Women taking any hormonal drugs and women of any chronic diseases were excluded in this study. A total of 211 women of childbearing age were included in the study. Sleep quality was assessed by using the PSQI. ${ }^{8}$ The PSQI is a self-rated instrument used to measure sleep quality in clinical samples to provide a reliable, valid, and standardized measure of sleep quality to discriminate between "good" and "poor" sleepers. Global PSQI score ranges from ' 0 ' to ' 21 '. PSQI score $\leq 5$ indicates good sleepers and PSQI score $>5$ indicates poor sleepers. The data were tabulated in MS Office Excel. Graphs were made using MS office. Statistical analysis was done SPSS software. Analysis of variance (ANOVA) test was applied for comparative analysis. $P$ value $<0.05$ was considered as significant.

\section{Results}

A total of 211 women of childbearing age were included in the study. They were between 15 years and 49 years with the mean age of $29.53 \pm 6.69$ years and mean body mass index (BMI) of $24.02 \pm 1.03$ $\mathrm{kg} / \mathrm{m}^{2}$. Graph 1 shows sleep quality according to different age
1.4 Senior Resident, ${ }^{2}$ Professor and Head, ${ }^{3} \mathrm{DM}$ fellow

Deparment of Physiology, MKCG Medical College, Berhampur, Odisha, India

2Department of Physiology, Government Medical College, Bolangir, Odisha, India

${ }^{3}$ Department of Pulmonary Medicine, All India Institute of Medical Science, New Delhi, India

${ }^{4}$ Department of Community Medicine, Srirama Chandra Bhanja Medical College, Cuttack, Odisha, India

Corresponding Author: Arpita Priyadarshini, Professor and Head, Department of Physiology, Government Medical College, Bolangir, Odisha, India, email-doctor@drarpita.in

Howtocitethis article:PattanaikA,PriyadarshiniA,VaidyaS, PattanaikN. Assessment of Sleep Quality Among Women of Childbearing Age by Pittsburgh Sleep Quality Index. Indian Sleep Med 2019;14(1):7-9

Source of support: Nil

Conflict of interest: None

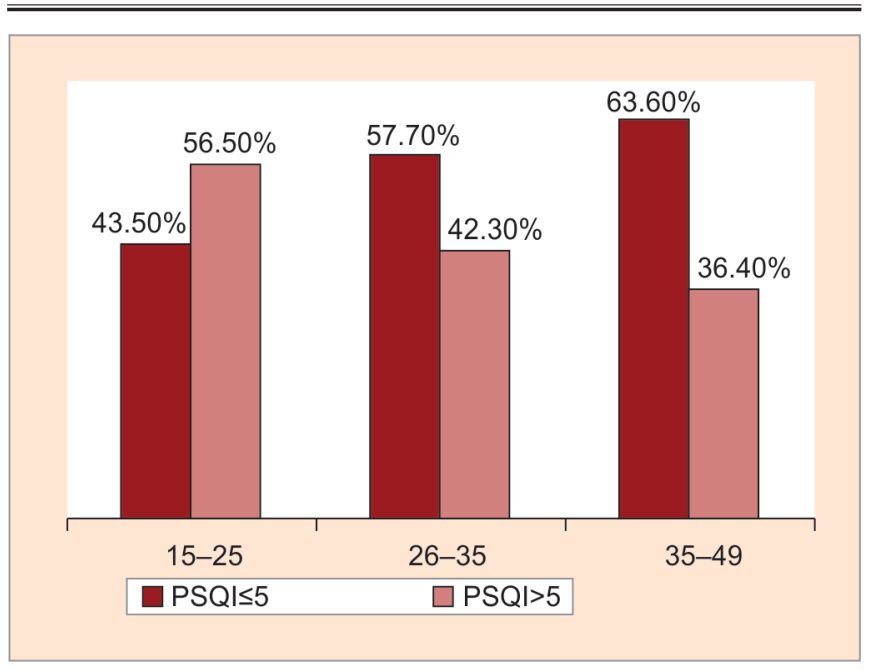

Graph 1: Age group vs. PSQI score

() The Author(s). 2019 Open Access This article is distributed under the terms of the Creative Commons Attribution 4.0 International License (http://creativecommons. org/licenses/by/4.0/), which permits unrestricted use, distribution, and non-commercial reproduction in any medium, provided you give appropriate credit to the original author(s) and the source, provide a link to the Creative Commons license, and indicate if changes were made. The Creative Commons Public Domain Dedication waiver (http://creativecommons.org/publicdomain/zero/1.0/) applies to the data made available in this article, unless otherwise stated. 


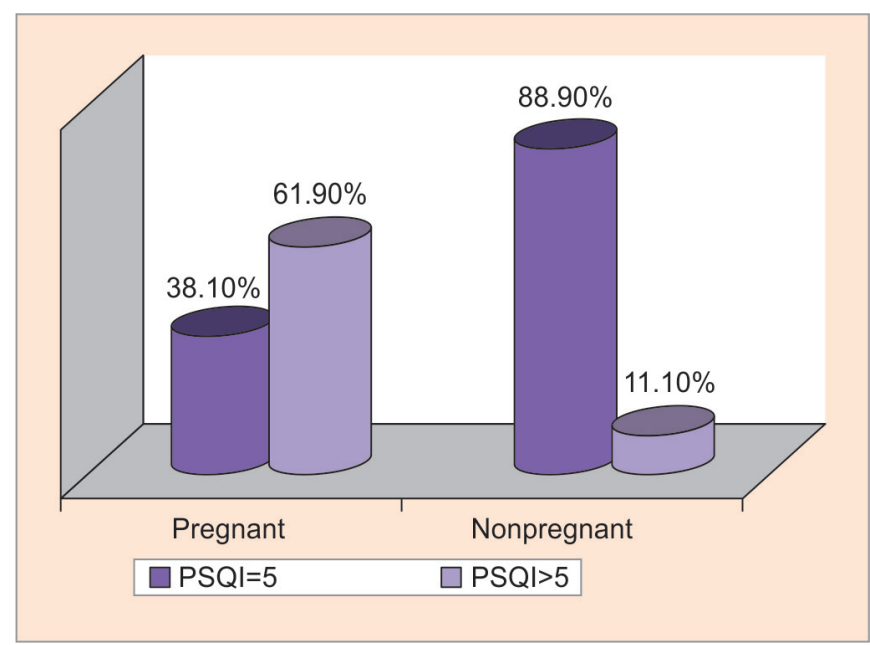

Graph 2: Global PSQI score with pregnant and nonpregnant status

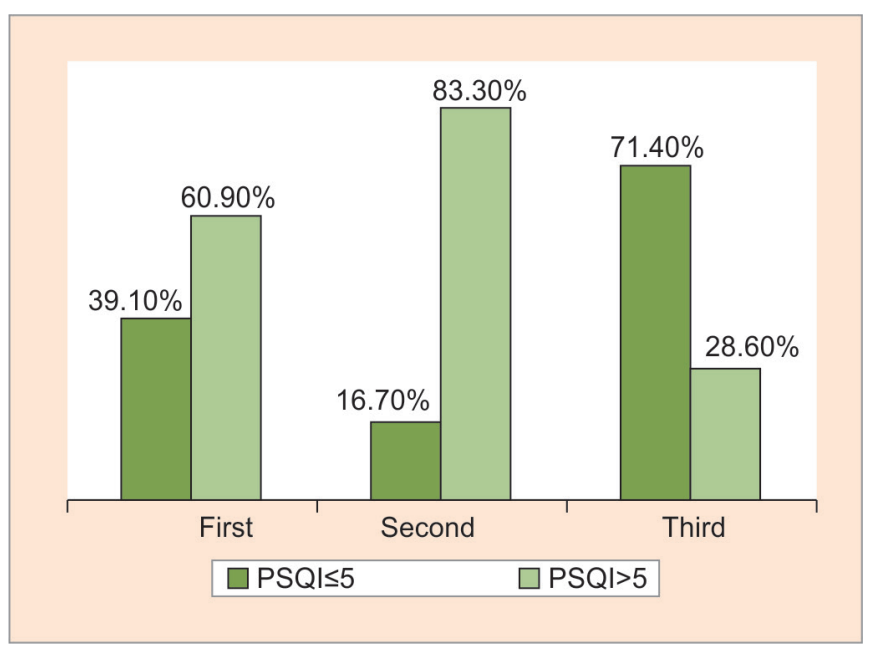

Graph 3: Trimsters of pregnancy vs. PSQI score groups. Nearly $43.5 \%$ of women were in the age group of $15-25$ years, $57.7 \%$ in the age group of $26-35$ years and $65.6 \%$ women in the age group of 35-49 years were good sleepers. Among poor sleeper, $56.5 \%$ of women were in the age group of $15-26$ years, $42.3 \%$ were in the age group of $26-35$ years, and $36.4 \%$ were in the age group of 35-49 years.

Global PSQI score with the pregnant and nonpregnant status of women is shown in Graph 2. About $38.1 \%$ of pregnant and $88.9 \%$ of non-pregnant women were good sleepers whereas $61.9 \%$ of pregnant and $11.9 \%$ of nonpregnant were poor sleepers. The difference was statistically significant $(p<0.05)$.

Graph 3 shows the PSQI score in the three trimesters of pregnancy with sleep. $39.1 \%$ in the first trimester, $16.7 \%$ in the second trimester and $71.4 \%$ in the third trimester were good sleepers. Among poor sleeper pregnant women $60.9 \%$ were from the first trimester, $83.3 \%$ from the second trimester and $28.6 \%$ were from the third trimester. The difference was statistically significant for pregnant women in the 2 nd trimester $(p<0.05)$.

\section{Discussion}

Pregnancy is related to multiple sleep disturbances. There can be many biological reasons for sleep disturbances associated with pregnancy, hormonal changes being the most common. ${ }^{9}$ Progesterone administration has resulted in changes in sleep cycle in animal studies. ${ }^{10}$ The common sleep problems reported in pregnancy include changes in duration and pattern of sleep, insomnia, restless leg syndrome, and snoring. ${ }^{9,11}$

In this study, 211 women of childbearing age (15-49 years) were taken as study subjects. Women taking any hormonal drugs and women of any chronic diseases were excluded from the study. $56.5 \%$ of women from age group 15-25 years had PSQI >5, i.e. poor sleepers which was highest from other age groups. Pucci et al. ${ }^{12}$ also got a similar finding. They concluded that sleep abnormalities start from early adulthood. This study also revealed that most of the pregnant (61.9\%) women were a poor sleeper in compare to nonpregnant women. This result is similar to the study conducted by Sahota et al. ${ }^{13}$ and Bourjeily et al. ${ }^{14}$ who also found that sleep disturbance was a common sleep abnormality in pregnancy. Among pregnant women, maximum sleep abnormalities were found in the second trimester (83.3\%) in comparison to another trimester of pregnancy. This result is in contrary to a study conducted by Sedov et al. ${ }^{15}$ which says maximum sleep abnormalities in the third trimester, next is second and least in the first trimester of pregnancy.

A future longitudinal study should be done to assess how this sleep quality change affects pregnancy.

Insomnia or excess sleepiness which develops during pregnancy is called pregnancy-associated sleep disorder. ${ }^{16}$ Patients should be advised on sleep hygiene techniques, and pregnancyrelated conditions should be addressed. A careful history is needed to identify specific sleep disorder, and patients can be subjected to further testing if needed. ${ }^{9}$

\section{Conclusion}

In this study, we found that sleep quality changes in women start at a very early age (15-25 years). Most women suffer from sleep problem during pregnancy among which maximum in the 2 nd trimester of pregnancy. So sleep quality management is very much essential in women particularly in pregnancy.

\section{Limitations of the Study}

As PQSI is a subjective questionnaire-based tool, sleep disorders were not objectively identified. Polysomnography could not be done. We could not make out the exact cause of poor sleep in pregnant patients as there are many causes for poor sleep in them.

\section{References}

1. Born J. Sleep and immune functions. In: Schedlowski M, Tewes $U$, editors. Psychoneuroimmunology: an Interdisciplinary Introduction. Kluwer Academic/Plenum Publishers; New York: 1999. pp. 417-442.

2. Alvarez GG, Ayas NT. The impact of daily sleep duration on health: a review of the literature. Prog Cardiovasc Nurs. 2004;19:56-59.

3. Moline M, Broch L, Zak R. Sleep problems across the life cycle in women. Current treatment options in neurology. 2004;6(4):319-330.

4. Mauri M. Sleep and the reproductive cycle: a review. Health Care Women Int. 1990:11(4):409-421.

5. http://www.who.int/reproductivehealth/topics/infertility/ definitions/en/cited on 02.07.2018.

6. Moline ML, Broch L, Zak R, Gross V. Sleep in women across the life cycle from adulthood through menopause. Sleep medicine reviews. 2003 Apr;7(2):155-177..

7. Irwin MR, Wang M, Campomayor CO, Collado-Hidalgo A, Cole S. Sleep deprivation and activation of morning levels of cellular and genomic markers of inflammation. Arch Intern Med. 2006;166:175-176. 
8. Buysse DJ, Reynolds III CF, Monk TH, Berman SR, Kupfer DJ. The Pittsburgh Sleep Quality Index: a new instrument for psychiatric practice and research. Psychiatry research. 1989;28(2):193-213.

9. Pien GW, Schwab RJ. Sleep disorders during pregnancy. Sleep. 2004;27(7):1405-417.

10. Lancel MA, Faulhaber JO, Holsboer FL, Rupprecht RA. Progesterone induces changes in sleep comparable to those of agonistic GABAA receptor modulators. American Journal of Physiology-Endocrinology and Metabolism. 1996;271(4):E763-772.

11. Facco FL, Kramer J, Ho KH, Zee PC, Grobman WA. Sleep disturbances in pregnancy. Obstet Gynecol. 2010;115(1):77-83.
12. PucciSH, Pereira MG. Sleep Quality in Adolescents:What's Discriminates Good from Poor Sleepers. J Sleep Disord Ther. 2016;5(237):2167-2277.

13. Sahota PK, Jain SS, Dhand R. Sleep disorders in pregnancy.Curr Opin Pulm Med. 2003;9(6):477-483.

14. Bourjeily, Ghada. "Sleep Disorders in Pregnancy." Obstetric Medicine 2.3 (2009): 100-106. PMC. Web. 2 July 2018.

15. Sedov ID, Cameron EE, Madigan S, Tomfohr-Madsen LM. Sleep Med Rev. 2018;38:168-176.

16. International Classification of Sleep Disorders, Revised: Diagnostic and Coding Manual. Rochester, MN: American Academy of Sleep Medicine; 2000:14-17.

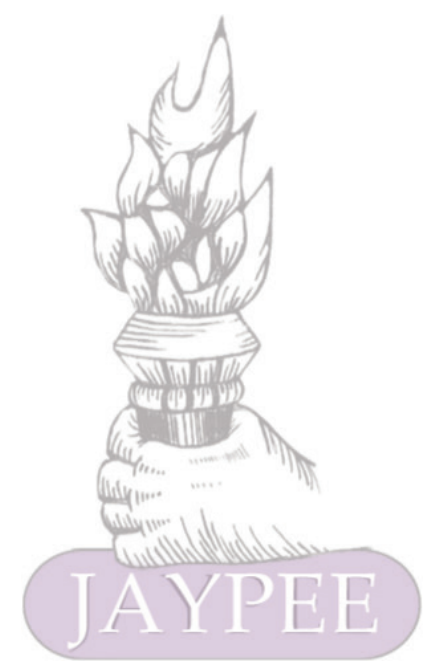

